\title{
Review on Basicervical Femoral Neck Fracture: Definition, Treatments, and Failures
}

\author{
Jun-Il Yoo, MD, Yonghan Cha, MD*, Joonhyeok Kwak, MD*, Ha-Yong Kim, MD*, Won-Sik Choy, MD* \\ Department of Orthopaedic Surgery, Gyeongsang National University Hospital, Jinju, Korea, \\ Department of Orthopaedic Surgery, Eulji University Hospital, Daejeon, Korea*
}

Basicervical femoral neck fracture is an uncommon fracture that accounts for only $1.8 \%$ of all proximal femoral fractures. Previous studies have recommended that the choice of implant to treat this fracture should be similar to that of intertrochanteric fracture. However, in previous studies on basicervical fractures, the definition and treatment results of these fractures were different, and there were also debates on the implant that had to be used. Therefore, the purpose of this study was to review the studies that performed surgical treatment of basicervical femoral fractures and to assess the definition of basicervical fracture, the use of implants, and failure rates and clinical results. Study selection was based on the following inclusion criteria: (1) treatment outcome for basicervical femoral neck fracture was reported; and (2) dynamic hip screw, proximal femoral nail, or multiple screw fixation was used as treatment. PubMed Central, OVID MEDLINE, Cochrane Collaboration Library, Web of Science, EMBASE, and AHRQ databases were searched to identify relevant studies published up to March, 2020 with English language restriction. A total of 15 studies were included in this study. Differences were found in the definition of basicervical fracture, treatment results, rehabilitation protocol, and fixation failure rate. Definitions and treatment methods for basicervical fractures varied, and treatment outcomes also differed among the enrolled studies. Further research is needed that would be restricted to those fractures that conform to the definition of basicervical fracture.

Key Words: Femoral neck fractures, Fracture fixation, Hip fracture, Internal

Submitted: March 24, 2020 1st revision: May 13, 2020

Final acceptance: May 15, 2020

Address reprint request to

Yonghan Cha, MD

(https://orcid.org/0000-0002-7616-6694)

Department of Orthopaedic Surgery, Eulji University College of

Medicine, 95 Dunsanseo-ro, Seo-gu, Daejeon 35233, Korea

TEL: +82-42-611-3280 FAX: +82-42-611-3283

E-mail: naababolahanmail.net

This is an Open Access article distributed under the terms of the Creative Commons Attribution Non-Commercial License (http://creativecommons. org/licenses/by-nc/4.0) which permits unrestricted non-commercial use, distribution, and reproduction in any medium, provided the original work is properly cited.

\section{INTRODUCTION}

Basicervical femoral neck fractures are rare and account for just $1.8 \%$ of all proximal femoral fractures ${ }^{1)}$. The line of a basicervical fracture goes through the base of the femoral neck at its junction with the intertrochanteric region ${ }^{2}$. Previous studies have recommended that the choice of implant to treat this fracture should be similar to that of intertrochanteric femoral fracture ${ }^{3)}$. However, due to the anatomical location of the fracture line, the proximal fragment in basicervical fractures has no muscular insertion, and unlike trochanteric fractures, these fractures lack cancellous interdigitation ${ }^{4}$. Thus, the basicervical femoral neck fractures are more instable than intertrochanteric fractures ${ }^{3)}$.

Recently, proximal femoral nails (PFNs) became widely 
used for treating intertrochanteric fractures ${ }^{5,6)}$. Various types of nail devices have been developed and used. However, Watson et al. ${ }^{7}$ have suggested that PFNs are unsuitable for treatment of basicervical femoral neck fractures. They reported that basicervical fractures healed without complications only in 5 among 11 patients, whereas 6 patients had fixation failure. In addition, their study did not support a suggestion that PFNs have biomechanical advantages over dynamic hip screws (DHSs). On the other hand, Hu et al. ${ }^{8}$ treated 32 patients with basicervical fractures using cephalomedullary hip nails, and there were no cut outs and clinical results were satisfactory.

To determine the best treatment strategy for basicervical fracture, we need to investigate the treatment methods and results of previous studies. However, even the same implant was used, different studies may reach different conclusions, which makes it difficult ascertain the choice of treatment option for these fractures. Also, the radiographs presented in some studies did not appear to match the definition of this fracture ${ }^{1,7,8)}$.

Therefore, the purpose of this study was to assess previous studies that used surgical treatment of basicervical femoral fractures to review the definition of a basicervical fracture, the type of treatment implants, failure rates, and clinical results.

\section{MATERIALS AND METHODS}

\section{Study Eligibility Criteria}

Study selection was based on the following inclusion criteria: (1) treatment outcomes (such as radiologic fracture healing period, Harris hip score [HHS], union rate, implant failure, non-union, reoperation) for a basicervical femoral neck fracture were reported; and (2) DHS, PFN, or multiple screw fixation was used as treatment. Studies were excluded if they failed to meet the above criteria.

\section{Search Methods for Identification of Studies}

PubMed Central, OVID MEDLINE, Cochrane Collaboration Library, Web of Science, EMBASE, and AHRQ databases were searched to identify relevant studies published up to March 2020, with English language restriction. The following search terms were used: "basicervical" [All Fields] AND ("fractures, bone" [MeSH Terms] OR ("fractures" [All Fields] AND "bone" [All Fields]) OR "bone fractures" [All Fields] OR "fracture" [All Fields]) (Appendix). Manual search was also conducted for possibly related references.
JIY, YC reviewed the titles, abstracts, and full texts of all potentially relevant studies independently, as recommended by the Cochrane Collaboration. Any disagreement was resolved by the fourth reviewer (HYK). We assessed the full text according to the inclusion criteria described above, and then selected eligible articles. The reviewers were not blinded to authors, institutions, or the publication.

\section{Data Extraction}

The following information was extracted from the included articles: authors, publication date, study design, study period, mean follow-up period, number of patients, mean age, definition of basicervical fracture, type of implants, outcome measurements, and ambulation protocol. Outcomes pooled in this analysis were radiologic fracture union time and HHS.

\section{RESULTS}

\section{Search Results}

The initial search identified 432 references. However, 407 studies were excluded after screening the abstracts and titles. The remaining 25 studies underwent full-text review. Ten studies were further excluded. Details on the identification of relevant studies are shown in the flow chart of the study selection process (Fig. 1). Study design, study period, and the number of patients included in our study are summarized in Table $1^{1,3,7-19)}$.

\section{Definition of Basicervical Fracture}

Definitions of basicervical fracture described in the articles included in this study are summarized in Table 2 . Basicervical femoral fractures in studies of Massoud ${ }^{3)}$, Chen et al. ${ }^{1)}$, and Kuokkanen ${ }^{13)}$ were defined as extracapsular fractures. Five studies defined basicervical fracture as a 2-part fracture ${ }^{7,11,14,15,17)}$. Davis et al. ${ }^{12)}$ did not mention the definition of a basicervical femoral fracture. Hu et al. ${ }^{8}$ defined basicervical fracture as a particular type of trochanteric fracture in which the fracture line can be seen radiologically to run across close to the base of the femoral neck and its junction with the intertrochanteric region. Yoo et al. ${ }^{19)}$ also mentioned that it is a variant of a trochanteric fracture and a partial capsular fracture.

However, contrary to the definitions of basicervical fracture in some studies, fracture line in the radiographs before 


\section{Hip \& Pelvis}

Hip Pelvis 32(4): 170-181, 2020

and after surgery presented in each study had a pattern involving the lesser or greater trochanter or disrupting the intertrochanteric line (Fig. 2) ${ }^{3,7,11-13)}$. The radiographs of basicervical fractures presented by Yoo et al. ${ }^{19)}$ and $\mathrm{Hu}$ et al. ${ }^{8)}$ clearly involved the intertrochanteric area, in agreement with their definitions of basicervical fractures as a variant of the intertrochanteric fractures (Fig. 3). In other studies, the presented radiographs show fractures that did not invade the intertrochanteric line and are 2-part fractures (Fig. 3) ${ }^{14-17}$.

\section{Implants}

Implants used for basicervical femoral neck fracture treatment in the identified studies are summarized in Table 3. Eleven studies used cephalomedullary nails were used $^{3,7-9,12,14-19)}$, and 8 studies used DHS ${ }^{1,3,10-13,15,18}$. Only 1 study used cancellous screws ${ }^{3)}$, and 5 studies compared different implants $\mathrm{s}^{3,12,15,18,19)}$. The types of cephalomedullary nails were a single screw, blade, and two integrated screws, and there were studies using additional anti-rotational cancellous screws for fixation with DHS. A very high failure rate was observed when cancellous screws were used ${ }^{3}$.

\section{Radiologic Fracture-healing Period, Harris Hip Score, and Ambulation}

Radiologic fracture-healing period, HHS, and ambulation status are summarized in Table 4. The reported healing period ranged from 8 to 24 weeks. Five studies that used HHS reported good results, with an average score of 80 or better ${ }^{1,3,9,16,17)}$. Different protocols were used to start ambulation. In the study of $\mathrm{Hu}$ et al. ${ }^{8}$, partial weight bearing was permitted at 8 weeks postoperatively. In the study of Massoud $^{3}$, patients were allowed to walk using crutches and toe touching until the absence of pain and a good callus was observed on radiographs.

\section{Treatment Results and Failures}

Treatment results, including failures, are summarized in

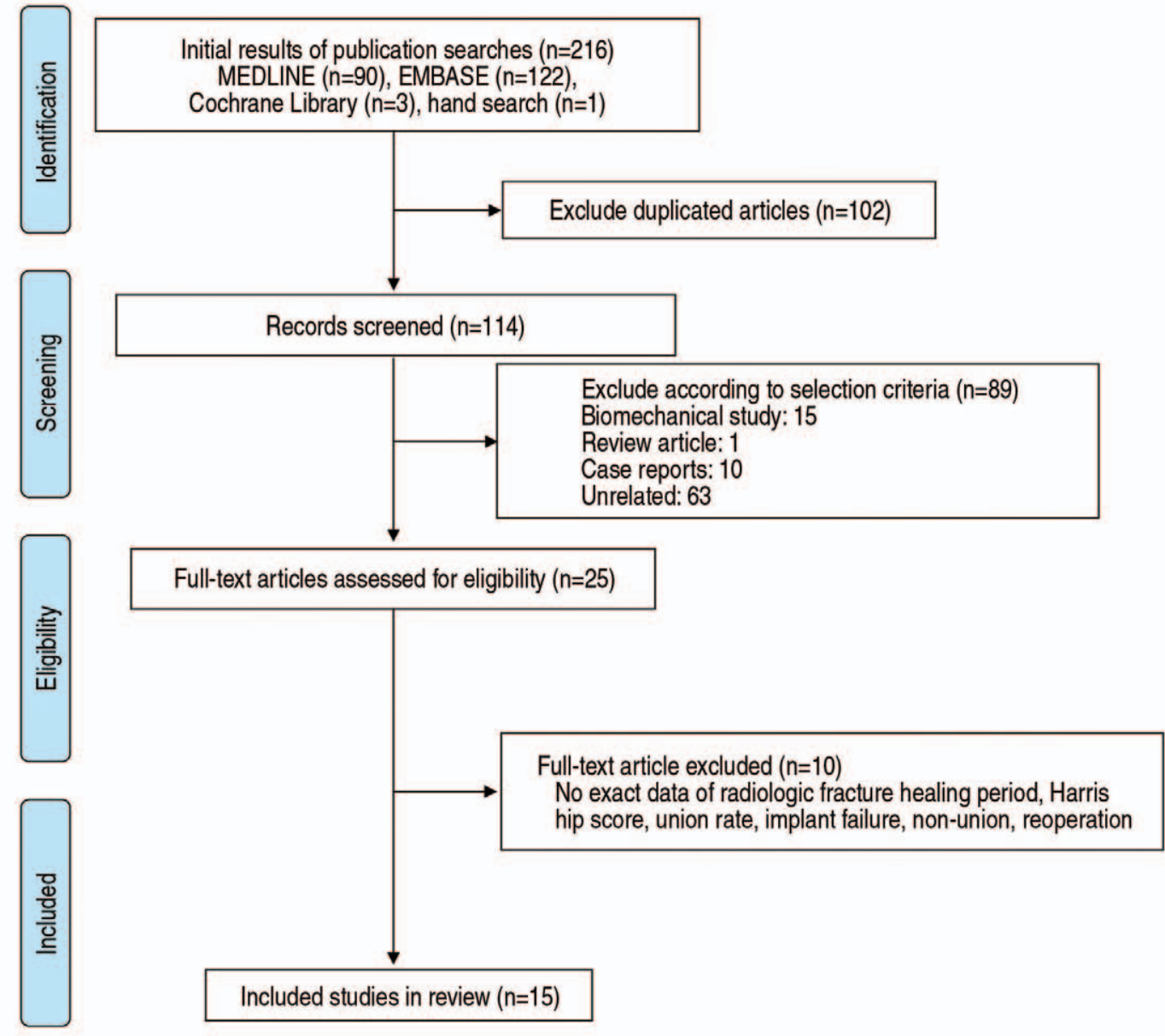

Fig. 1. The flow chart of the study selection process. 
Jun-Il Yoo et al. Review on Basicervical Femoral Neck Fracture

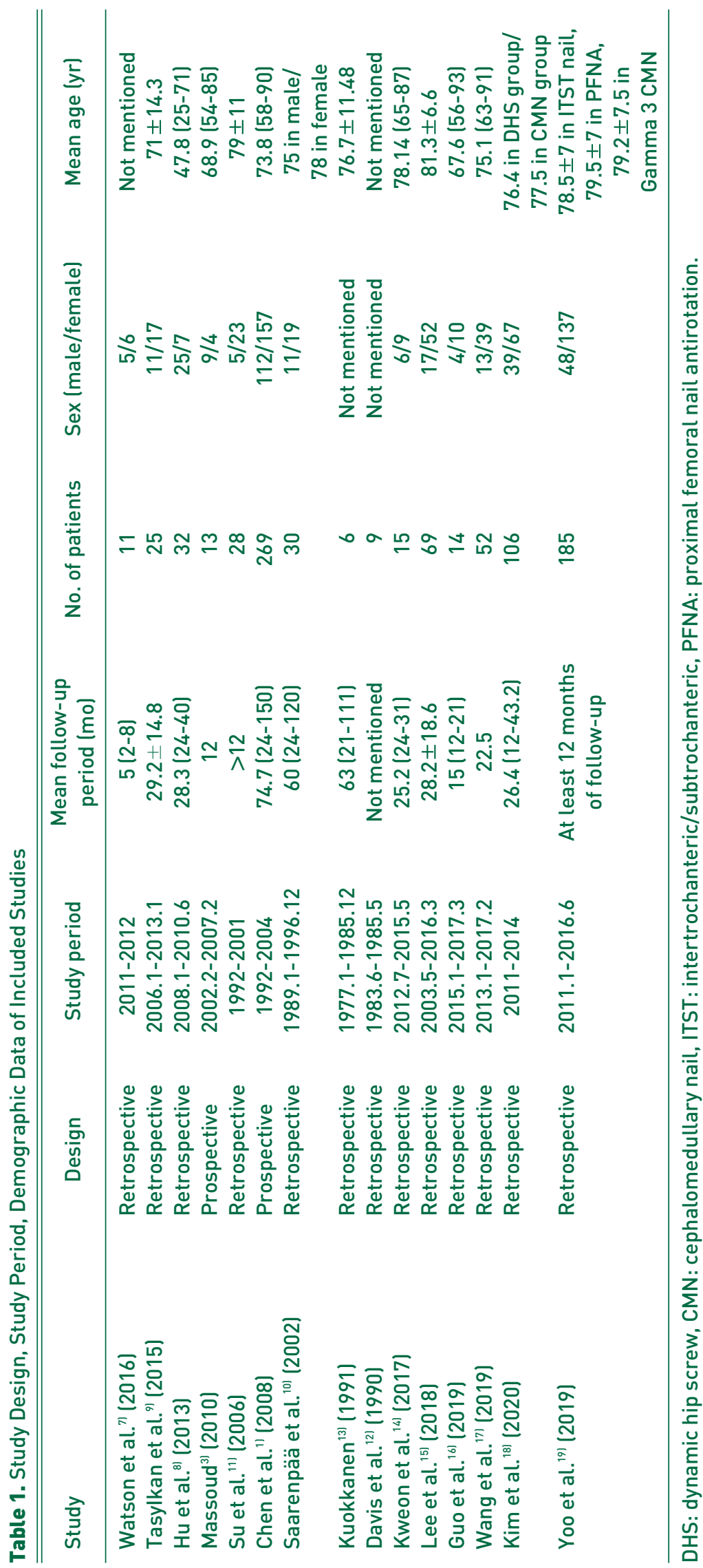

Table 5. Only 7 studies did not clearly define fracture collapse and failure of fixation ${ }^{3,7,11,15-17,19)}$. Watson et al.? reported fracture union rate (45\%), implant failure rate $(45 \%)$, and nonunion rate (5\%). Su et al. ${ }^{11)}$ reported that $75 \%$ patients had severe collapse. In a study by Lee et al. ${ }^{15}$, collapse occurred in $26.1 \%$ of the patients and the cut-out rate was $8.6 \%$. On the other hand, there were no collapse, nonunion, or total hip arthroplasty conversion cases in 6 studies s, $8,9,14,16,17)^{\text {. }}$

\section{DISCUSSION}

Although the studies included in this study had similar definitions regarding where the line of the basicervical fracture is located in the medial of the intertrochanteric line, the definitions of the basicervical fractures differed among the studies in whether they are accompanied by comminution or are extra- or intracapsular fracture. In addition, radiographs presented in each study were inconsistent with the definition of basicervical fractures in the same study. Due to this, the proportion of basicervical fractures among all hip fractures varies among the studies. Kim et al. ${ }^{18}$ reported a prevalence of basicervical fractures of $4.5 \%$. In the study of Lee et al. ${ }^{15}$, $2.4 \%$ of hip fractures were basicervical fractures. On the other hand, in the study of Guo et al. ${ }^{16}$, only 17 out of 2,291 femoral neck fracture patients had basicervical fractures $(0.74 \%)$. It is difficult to provide the exact definition and location of the basicervical fracture using the previously used fracture classification method ${ }^{10,20}$. Considering the extracapsular location, large fracture angle, and the absence of muscular attachment to the proximal fragment, basicervical fractures have to be classified as $\mathrm{AO}$ type $\mathrm{B} 2.1^{2,3,10,21)}$. Other studies have reported that AO type A2.1, A2.2, and A2.3 or Jensen type 3-5 with rotational instability of the headneck fragment or Jensen type 1-2 are similar to each other ${ }^{22-24}$. Although many studies have evaluated the fracture type by using simple radiographs, three-dimensional computed tomography might be necessary for accurate diagnosis ${ }^{10,25}$. The radiographs presented in 
Hip Pelvis 32(4): 170-181, 2020

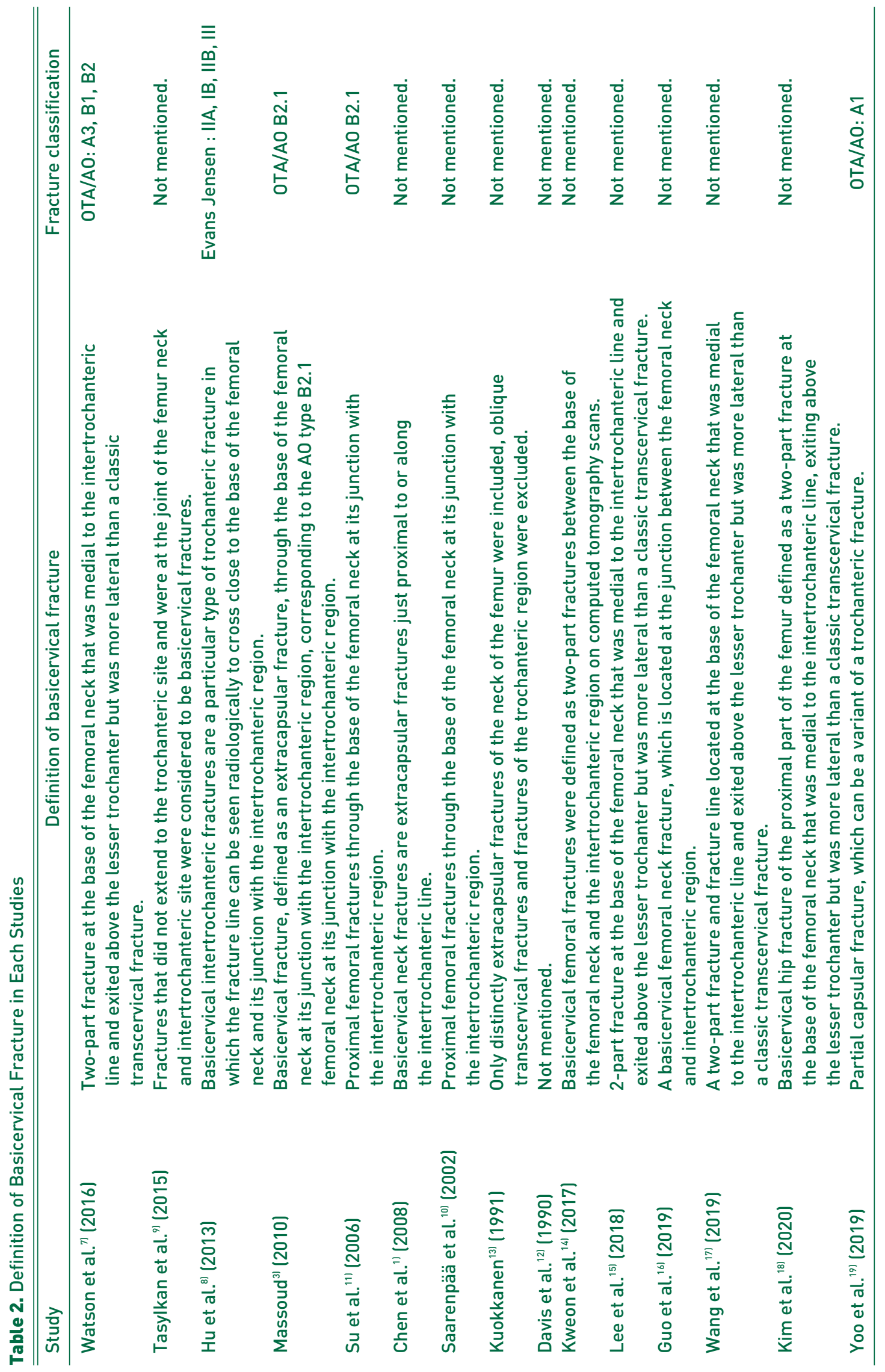



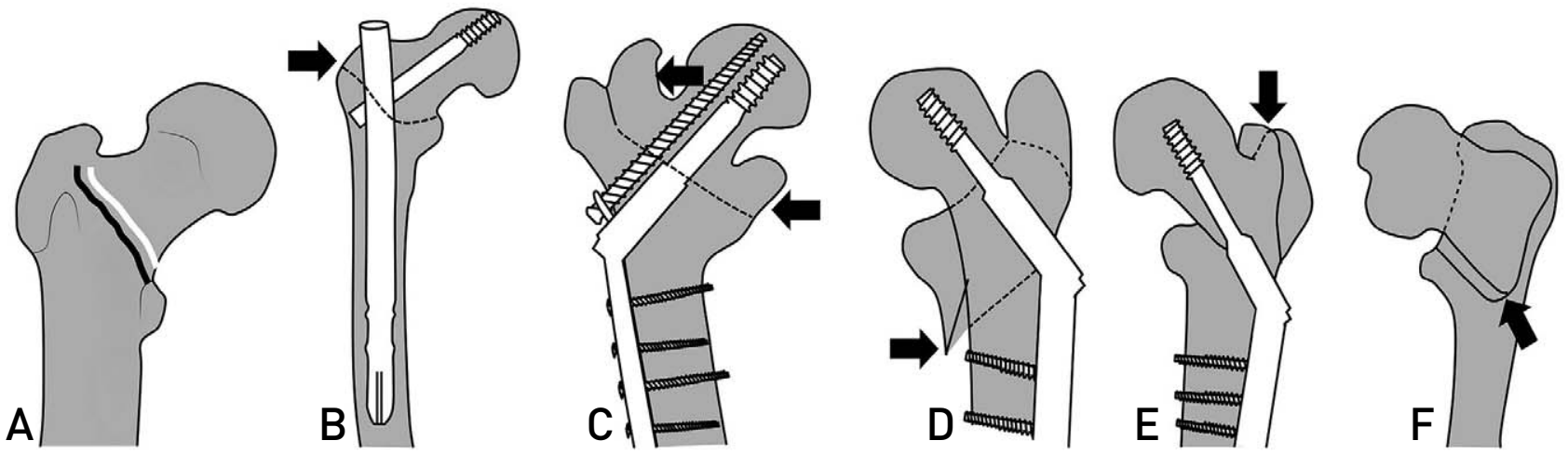

Fig. 2. The radiographs in included studies. (A) Normal bony structure of the proximal femoral head. The intertrochanteric line is shown in black and the line of the basicervical fracture in white. (B) Schematic figure depicting a radiograph in the study of Watson et al. ${ }^{71}$. The fracture line started in the inferior area of the intertrochanteric line (black arrow). (C) Schematic figure showed the radiograph in study of Massoud. ${ }^{3)}$ The fracture line crossed the intertrochanteric line (black arrow). (D) Schematic figure depicting the radiograph in the study of Davis et al. ${ }^{12 l}$ The lesser trochanteric area was involved in the fracture (black arrow). (E) Schematic figure depicting the radiograph in the study of Kuokkanen. ${ }^{13)}$ Fracture involved the greater trochanter (black arrow). (F) Schematic figure depicting the radiograph in the study of Su et al. ${ }^{11}$. The inferior area of the fracture line involved the intertrochanteric line (black arrow). Fractures not matching the definition of basicervical fracture.
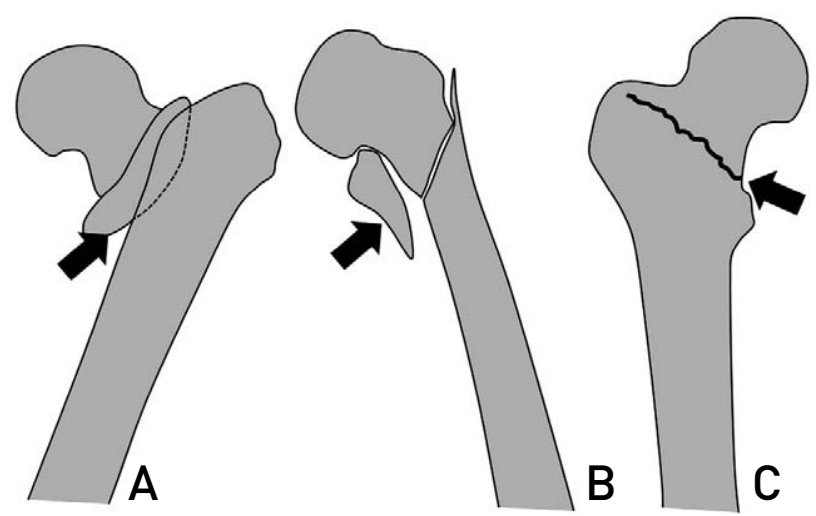

Fig. 3. The radiographs in included studies. (A) Schematic figure depicting the radiograph in the study of $\mathrm{Hu}$ et $\mathrm{al}^{{ }^{81}}$. Proximal fragment included part of the lesser trochanter (black arrow). (B) Schematic figure depicting the radiograph in the study of Yoo et al. ${ }^{191}$. The lesser trochanter fragment is displaced (black arrow). (C) Schematic figure depicting the radiograph in the study of Lee et al. ${ }^{15)}$. They showed radiograph of a 2-part basicervical fracture (black arrow).

each study differ in the type of implant, fracture union, and fixation failure, but the fracture line consistently disrupts the intertrochanteric line or involves the lesser trochanter and greater trochanter. Because of this, patients with intertrochanteric fractures were enrolled in each study, which make the results of treatment difficult to interpret.

The types of implants used for fixation of the basicervical fractures were very diverse, and treatment results such as fixation failure rate and collapse rate of the fracture site were also very different. It remains difficult to fully trust the results of each study or to discuss the superiority or the effectiveness of a particular implant, because the question remains as to whether the subjects enrolled in each study met the definition of basicervical fracture. Femoral neck fractures lead to rotational instability of the proximal fragment ${ }^{22,26,27}$. Therefore, the single-screw type of cephalomedullary fixation dose not achieve accurate reduction or stable fixation. On the other hand, an intertrochanteric fracture has more rotational stability than a femoral neck fracture. However, there is still controversy on rotational instability of the proximal fragment in patients with basicervical fractures in which the head-neck fragment does not involve the trochanteric area with a long inferior cortical extension. On the basis of biomechanical test for the basicervical fracture, Blair et al. ${ }^{2)}$ have reported no difference in torsional stability between a sliding hip screw alone and a sliding hip screw combined with an anti-rotational cancellous screw. However, Massoud $^{3)}$ have suggested that an anti-rotational screw should be added to control rotational instability after surgery because the lag screw has potential to rotate the rotationally unstable femoral head during its insertion. Authors of recent reports included in this study argue that the antirotational effect of implant should be taken into account when selecting an implant for the fixation of these rotationally unstable fractures ${ }^{14-19}$.

The main variables for radiological evaluation of basicervical fractures were the presence of collapse, cut-out of the screw, and nonunion. Each study had a different view on whether collapse should be interpreted as failure. Su et al. ${ }^{11}$ 


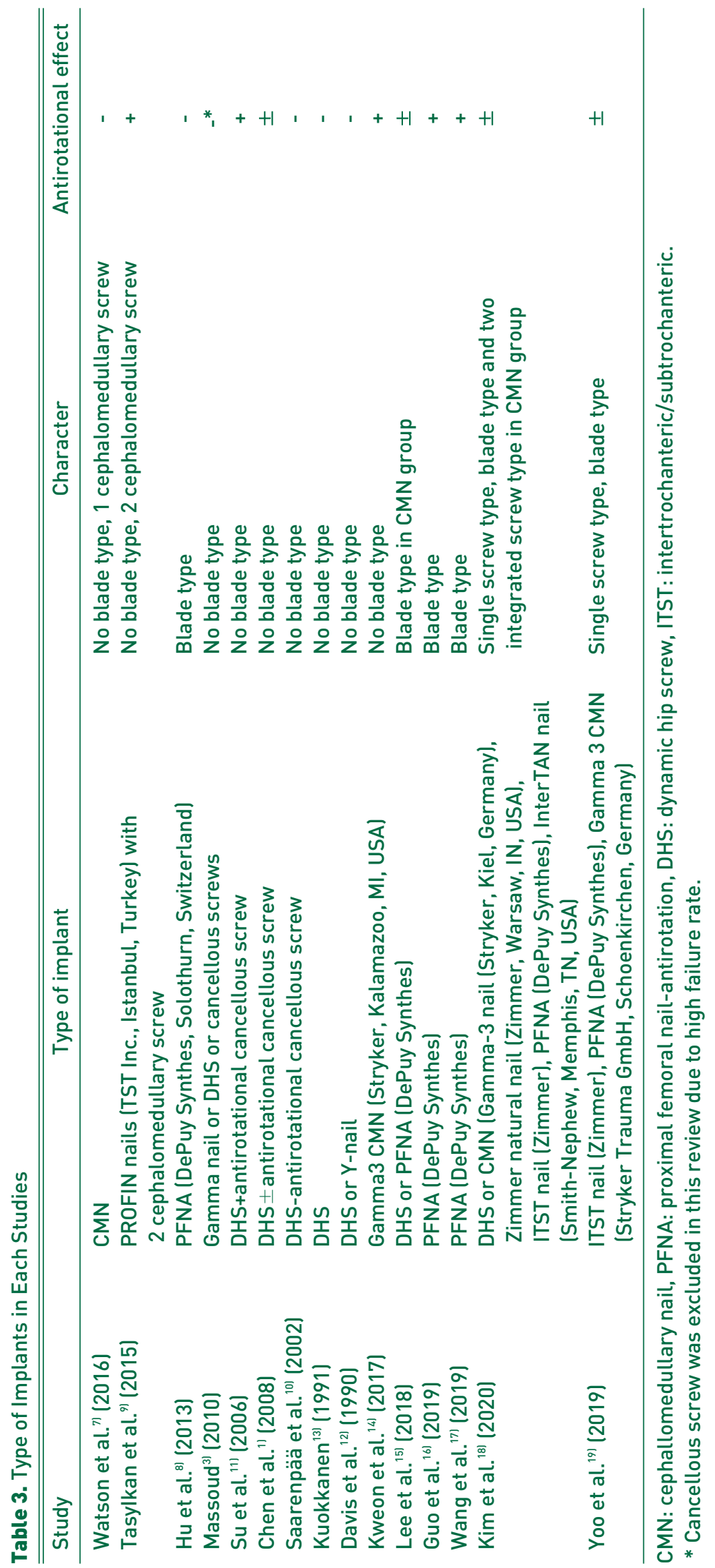

did not define collapse as a fixation failure. Although the rate of collapse was very high $(75 \%)$ in their study due to the protrusion of a compression screw, only three cases had revision due to fixation failure associated with varus malunion. There was no evidence to clinically determine the failure because functional assessment of patients with severe collapse was not reported separately. Varus collapse was also observed by Tasylkan et al. ${ }^{9)}$ and $\mathrm{Hu}$ et al. ${ }^{8}$, but was not included in the failure category. Tasylkan et al. ${ }^{9}$ did not include varus unions of 10-20 degrees in the failure category either. However, Watson et al." did include collapse in the definition of fixation failure. They reported a much higher failure rate than in other studies. In addition, problems such as short follow-up period, selection bias, and lack of analysis of cephalomedullary screw position might have caused their high failure rate.

Some studies described collapse and varus as fixation failure. Although there was no definitive evidence of rotational stability of the proximal fragment in patients with basicervical femoral neck fractures, there was no difference in results between patients treated with DHS only and those treated with DHS and an antirotation cancellous screw. There was no difference in torsional stability in a mechanical test either between DHS and DHS with a cancellous screw ${ }^{2}$. The main mechanism of failure seemed to be collapse to varus or a slide to the axis of the cephalomedullary screw ${ }^{2,11,12,28)}$. The shape of the section of the basicervical femoral neck is oval and does not rotate easily in the distal fragment. Because the proximal part of the fracture site is smaller than the distal part, the possibility of impaction of the proximal part into the distal part of the marrow is high. Su et al. ${ }^{11)}$ found a high rate of sliding of the cephalomedullary screw. Therefore, anatomical structure appears to contribute to failure. In addition, when a proximal cephalomedullary nail was used, the proximal fragment might have unevenly slide, leading to varus collapse. This seems to be a likely mechanism of a high failure rate in Watson et al.'s study ${ }^{7}$. However, factors affecting collapse of a fracture site, such as reduction quality, 
Jun-Il Yoo et al. Review on Basicervical Femoral Neck Fracture

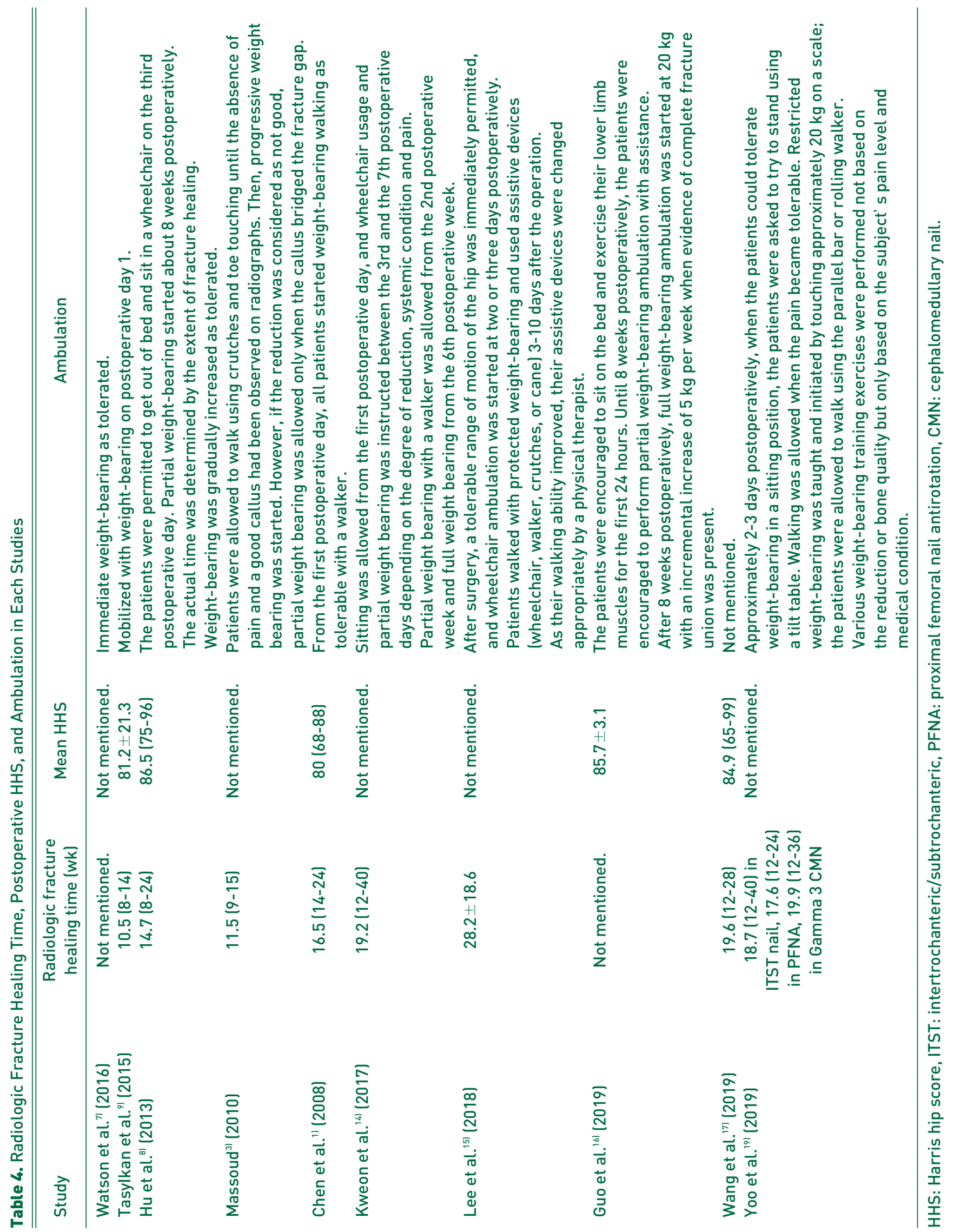




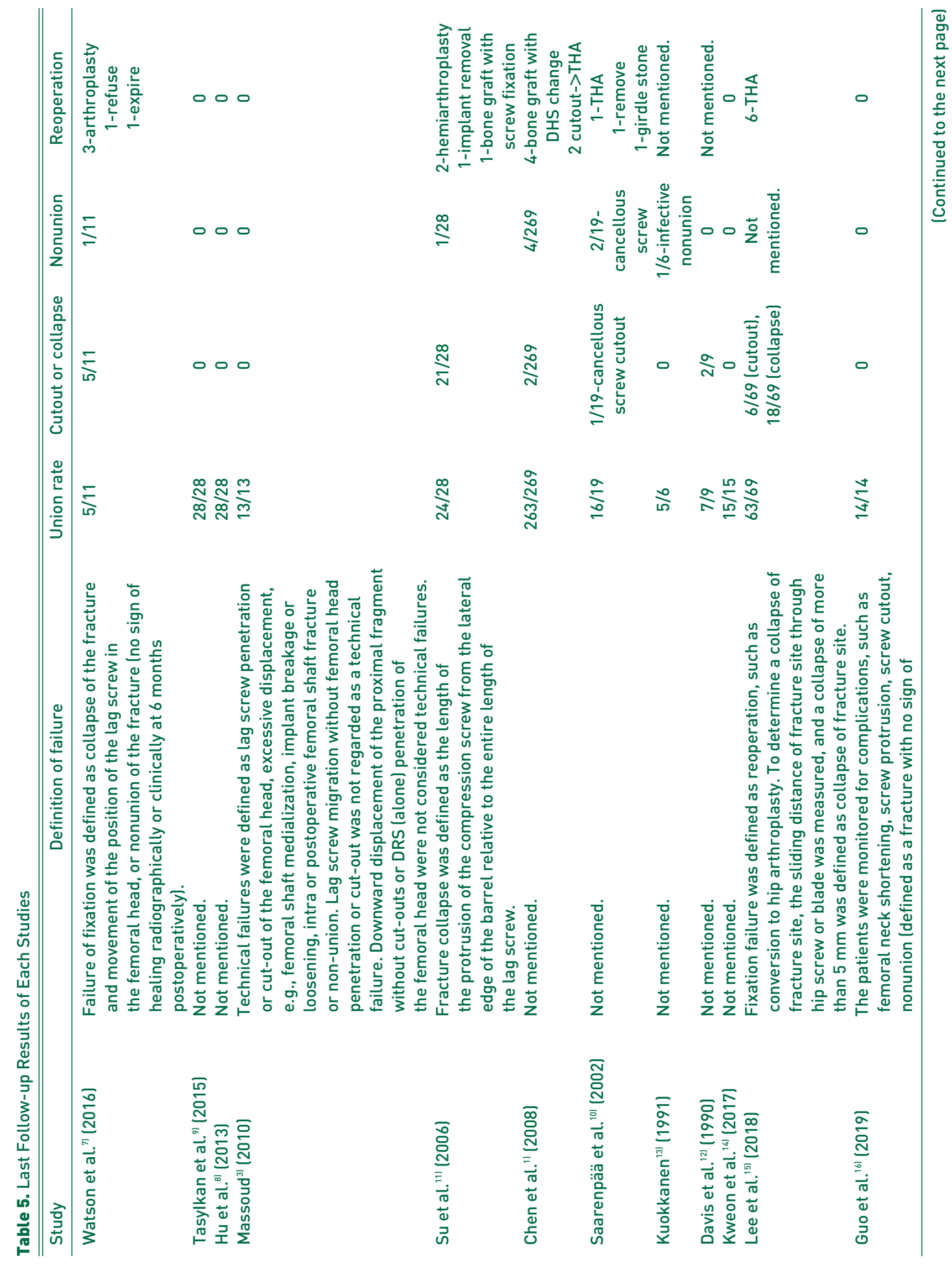


Jun-Il Yoo et al. Review on Basicervical Femoral Neck Fracture

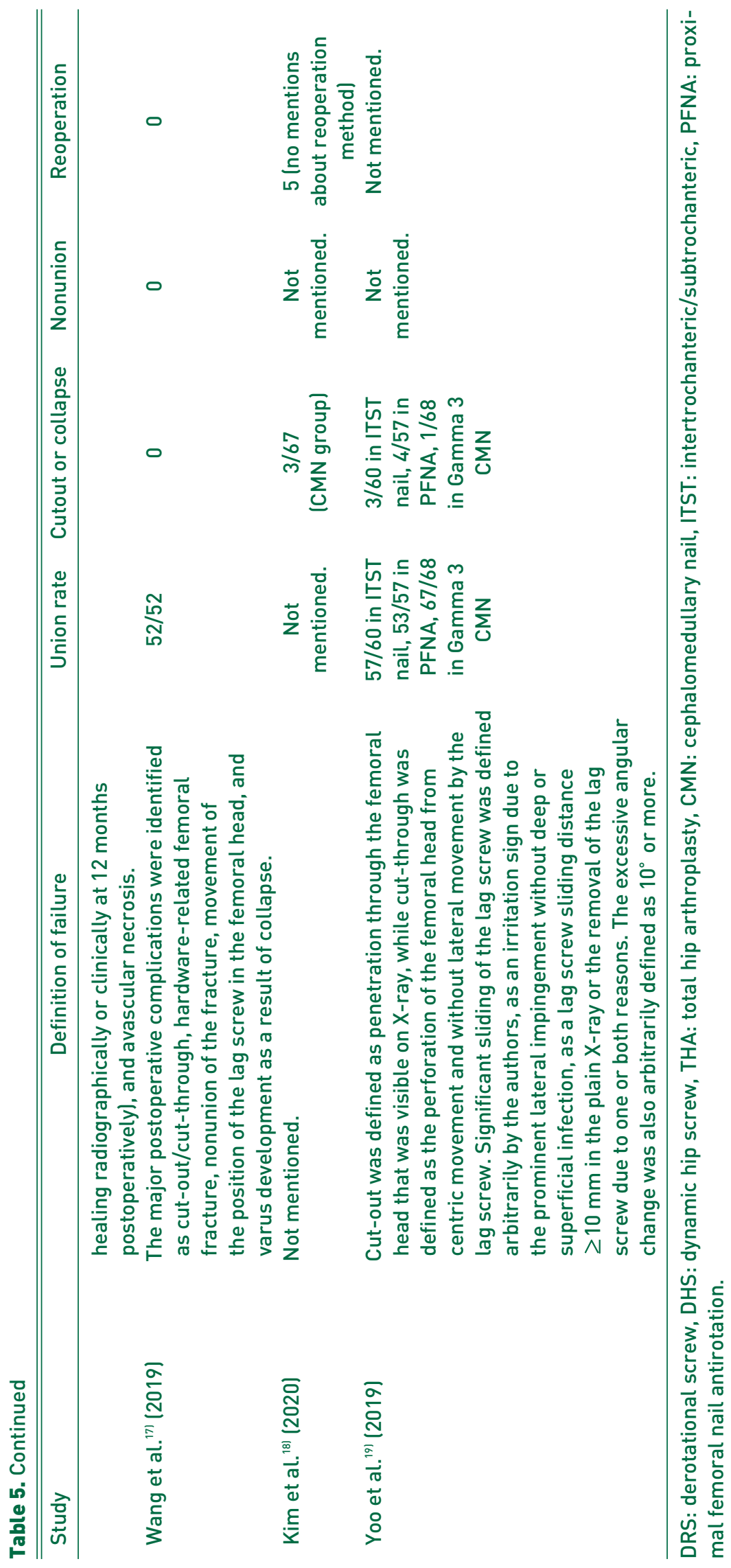

tip-apex distance, insertion position of the cephalomedullary screw, and time of weight bearing might vary ${ }^{1,7}$. Some studies showed good results after treatment of basicervical fractures with cephalomedullary nails ${ }^{9,10}$. Further studies on the treatment outcomes in patients with basicervical fractures treated by using PFNs are needed.

A limitation of this study was that metaanalysis of treatment results or fixation failure for each implant was not possible due to the heterogeneity of the included reports.

\section{CONCLUSION}

Definitions of and treatment methods for basicervical fractures varied, and treatment outcomes also differed among the enrolled studies. Further research is needed that would be restricted to those fractures that conform to the definition of basicervical fracture.

\section{CONFLICT OF INTEREST}

The authors declare that there is no potential conflict of interest relevant to this article. 


\section{REFERENCES}

1. Chen CY, Chiu FY, Chen CM, Huang CK, Chen WM, Chen TH. Surgical treatment of basicervical fractures of femur--a prospective evaluation of 269 patients. J Trauma. 2008;64: 427-9.

2. Blair B, Koval KJ, Kummer F, Zuckerman JD. Basicervical fractures of the proximal femur. A biomechanical study of 3 internal fixation techniques. Clin Orthop Relat Res. 1994; (306):256-63.

3. Massoud EI. Fixation of basicervical and related fractures. Int Orthop. 2010;34:577-82.

4. Imren Y, Gurkan V, Bilsel K, et al. Biomechanical comparison of dynamic hip screw, proximal femoral nail, cannulated screw, and monoaxial external fixation in the treatment of basicervical femoral neck fractures. Acta Chir Orthop Traumatol Cech. 2015;82:140-4.

5. Higgins JP, Thompson SG, Deeks JJ, Altman DG. Measuring inconsistency in meta-analyses. BMJ. 2003;327:557-60.

6. Stang A. Critical evaluation of the Newcastle-Ottawa scale for the assessment of the quality of nonrandomized studies in meta-analyses. Eur J Epidemiol. 2010;25:603-5.

7. Watson ST, Schaller TM, Tanner SL, Adams JD, Jeray KJ. Outcomes of low-energy basicervical proximal femoral fractures treated with cephalomedullary fixation. J Bone Joint Surg Am. 2016;98:1097-102.

8. Hu SJ, Yu GR, Zhang SM. Surgical treatment of basicervical intertrochanteric fractures of the proximal femur with cephalomeduallary hip nails. Orthop Surg. 2013;5:124-9.

9. Tasyıkan L, Ugutmen E, Sanel S, Soylemez MS, Ozkan K, Solakoglu C. Short-term results of surgical treatment with cephalomedullary nails for basicervical proximal femoral fractures. Acta Orthop Belg. 2015;81:427-34.

10. Saarenpää I, Partanen J, Jalovaara P. Basicervical fracture--a rare type of hip fracture. Arch Orthop Trauma Surg. 2002;122: 69-72.

11. Su BW, Heyworth BE, Protopsaltis TS, et al. Basicervical versus intertrochanteric fractures: an analysis of radiographic and functional outcomes. Orthopedics. 2006;29:919-25.

12. Davis TR, Sher JL, Horsman A, Simpson M, Porter BB, Checketts RG. Intertrochanteric femoral fractures. Mechanical failure after internal fixation. J Bone Joint Surg Br. 1990; 72:26-31.

13. Kuokkanen HO. Treatment options for basicervical fractures of the femoral neck. A clinical follow-up. Acta Orthop Belg. 1991;57:162-8.

14. Kweon SH, Lee SH, Kook SH, Choi YC. Outcomes of cephalomedullary nailing in basicervical fracture. Hip Pelvis. 2017;29:270-6.

15. Lee YK, Yoon BH, Hwang JS, Cha YH, Kim KC, Koo KH. Risk factors of fixation failure in basicervical femoral neck fracture: which device is optimal for fixation? Injury. 2018;49:691-6.

16. Guo J, Dong W, Jin L, et al. Treatment of basicervical femoral neck fractures with proximal femoral nail antirotation. J Int Med Res. 2019;47:4333-43.

17. Wang Q, Gu XH, Li X, et al. Management of low-energy basicervical proximal femoral fractures by proximal femoral nail anti-rotation. Orthop Surg. 2019;11:1173-9.

18. Kim JT, Ha YC, Park CH, Yoo JI, Kim TY. Single screw type of lag screw results higher reoperation rate in the osteosynthesis of basicervical hip fracture. J Orthop Sci. 2020;25: 152-5.

19. Yoo J, Kim S, Choi J, Hwang J. Gamma 3 U-Blade lag screws in patients with trochanteric femur fractures: are rotation control lag screws better than others? J Orthop Surg Res. 2019; 14:440.

20. Deneka DA, Simonian PT, Stankewich CJ, Eckert D, Chapman JR, Tencer AF. Biomechanical comparison of internal fixation techniques for the treatment of unstable basicervical femoral neck fractures. J Orthop Trauma. 1997;11:337-43.

21. Kyle RF, Gustilo RB, Premer RF. Analysis of six hundred and twenty-two intertrochanteric hip fractures. J Bone Joint Surg Am. 1979;61:216-21.

22. Swiontkowski MF. Intracapsular fractures of the hip. J Bone Joint Surg Am. 1994;76:129-38.

23. Pervez H, Parker MJ, Pryor GA, Lutchman L, Chirodian N. Classification of trochanteric fracture of the proximal femur: a study of the reliability of current systems. Injury. 2002; 33:713-5.

24. Jensen JS. Classification of trochanteric fractures. Acta Orthop Scand. 1980;51:803-10.

25. Shoda E, Kitada S, Sasaki Y, et al. Proposal of new classification of femoral trochanteric fracture by three-dimensional computed tomography and relationship to usual plain X-ray classification. J Orthop Surg (Hong Kong). 2017;25:2309499017692700.

26. Ly TV, Swiontkowski MF. Management of femoral neck fractures in young adults. Indian J Orthop. 2008;42:3-12.

27. Smith FB. Effects of rotatory and valgus malpositions on blood supply to the femoral head; observations at arthroplasty. J Bone Joint Surg Am. 1959;41:800-15.

28. Ort PJ, LaMont J. Treatment of femoral neck fractures with a sliding compression screw and two Knowles pins. Clin Orthop Relat Res. 1984;(190):158-62. 


\section{Hip\& Pelvis}

Jun-Il Yoo et al. Review on Basicervical Femoral Neck Fracture

Appendix. Detailed Search Strategies for Each Database

\begin{tabular}{|c|c|c|}
\hline Database & Detailed search strategies & Records founded \\
\hline MEDLINE/PubMed & $\begin{array}{l}\text { basicervical [All Fields] AND (“fractures, bone” [MeSH Terms] OR } \\
\text { ["fractures" [All Fields] AND “bone" [All Fields]) OR “bone fractures” } \\
\text { [All Fields] OR “fracture"[All Fields]) }\end{array}$ & 90 \\
\hline EMBASE & ‘basicervical fracture’ OR (basicervical AND ('fracture'/exp OR fracture)) & 122 \\
\hline $\begin{array}{l}\text { Cochrane Central Register } \\
\text { of Controlled Trials }\end{array}$ & $\begin{array}{l}\text { basicervical [All Fields] AND (“fractures, bone" [MeSH Terms] OR } \\
\text { ("fractures” [All Fields] AND “bone” [All Fields]) OR “bone fractures” } \\
\text { [All Fields] OR “fracture"[All Fields]) }\end{array}$ & 3 \\
\hline
\end{tabular}

Mesh terms, search terms, and combinations of the two were used for each database search. Ultimately, 216 records were found, 90 from MEDLINE/PubMed, 122 from EMBASE, and 3 from the Cochrane Library, and 1 from the hand search. Studies were further selected according to the inclusion criteria listed in the Material and Methods (Fig. 1). 